\title{
Comparison of Sexual Risk, HIV/STI Prevalence and Intervention Exposure Among Men Who Have Sex with Men and Women (MSMW) and Men Who Have Sex with Men Only (MSMO) in India: Implications for HIV Prevention
}

\author{
Lakshmi Ramakrishnan $^{1}$ (D) Shreena Ramanathan ${ }^{2} \cdot$ Venkatesan Chakrapani $^{3}$. \\ Prabuddhagopal Goswami $^{3}$ - Sucheta Deshpande ${ }^{4} \cdot$ Diwakar Yadav $^{1}$. \\ Shrabanti Sen ${ }^{2} \cdot$ Bitra George $^{2} \cdot$ Ramesh Paranjape $^{4}$ \\ Published online: 17 April 2015 \\ (C) The Author(s) 2015. This article is published with open access at Springerlink.com
}

\begin{abstract}
Using data from a cross-sectional bio-behavioral survey conducted among men who have sex with men $(n=3833)$ in India, we examined differences related to HIV-related sexual risk, HIV/STI prevalence and intervention exposures between men who have sex with men and women (MSMW, $35 \%$ of the sample) and men who have sex with men only (MSMO). Among MSMW, $93 \%$ reported having female regular partners, $14 \%$ had female paid partners, and all types of male partners (regular $55 \%$; casual $77.1 \%$; paying $47 \%$; paid $19 \%$ ). Logistic regression revealed that MSMW had higher odds of being aged 26 years and above (AOR 4.45, $95 \%$ CI 3.66-5.42), lower odds of inconsistently using condoms with male partners (AOR 0.82, $95 \%$ CI 0.67-0.98) and lower odds of having kothi (feminine/mostly receptive) identity (AOR 0.07, $95 \%$ CI 0.06-0.09). HIV intervention exposure and HIV/ STI prevalence did not differ significantly between MSMW
\end{abstract}

Lakshmi Ramakrishnan is formerly with FHI 360.

Shreena Ramanathan, Venkatesan Chakrapani, and Prabuddhagopal Goswami have contributed equally to this work.

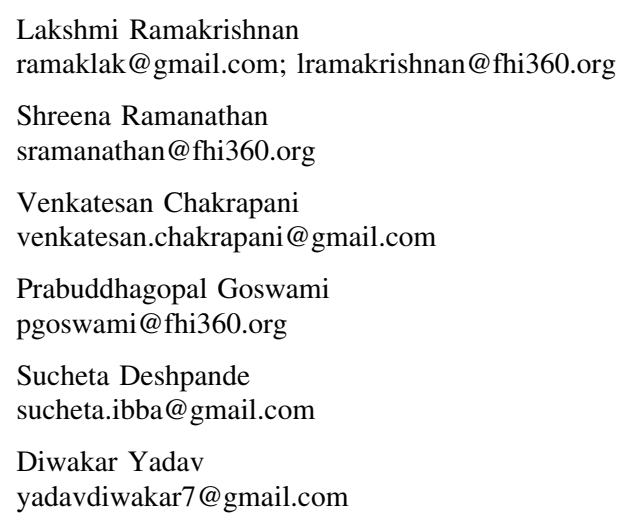

and MSMO (HIV 13.1 vs. $12.2 \%$; active syphilis 3.5 vs. $3.1 \%$, respectively). Concurrent sexual partnerships with men and women pose risk of HIV transmission/acquisition for MSM and their male and female partners. All subgroups of MSM require tailored information and skills to consistently use condoms with different types of partners of either gender.

Keywords Bisexual behavior - Men who have sex with men - Men who have sex with men and women - India . HIV · Condom use · Bisexual concurrency

\section{Introduction}

Globally, men who have sex with men (MSM) have significantly high HIV prevalence, due to structural and individual level vulnerabilities [1]. In India too, MSM are at a higher risk of HIV infection, with a national average HIV prevalence of $4.4 \%$ compared to $0.27 \%$ among the general population [2]. The term 'men who have sex with

\section{Bitra George}

bgeorge@ fhi360.org

Ramesh Paranjape

rparanjape@nariindia.org

1 No 11 Avatar Apartments, 27 Balakrishnan Road,

Valmikingar, Thiruvanmiyur, Chennai 600041, India

2 FHI 360 India, New Delhi, India

3 Centre for Sexuality and Health Research and Policy (C-SHaRP), Chennai, India

4 National AIDS Research Institute, Pune, Maharashtra, India 
men', in general, refers to any men who have sex with other men, regardless of their sexual orientation or sexual identity, whether or not they also have sex with women. However, for the purpose of this paper, the terms 'men who have sex with men only' (MSMO) and 'men who have sex with men and women' (MSMW) are used because the differences between these two groups may offer insights into differential sexual risks and HIV prevention targeting.

Among MSM from lower socioeconomic class, across India, certain identities such as kothi, double-decker and panthi are more common than gay or bisexual identities. Kothi refers to those same-sex attracted males who are feminine and primarily adapt receptive sexual role, doubledecker refers to those who adapt insertive or receptive sexual role, and panthi refers to masculine men who primarily adapt insertive sexual role [3, 4]. There are minor regional variations in this terminology. For example, instead of 'panthi', the terms 'parikh' or 'giriya' are used in certain parts of North India, and instead of 'double-decker', the terms 'do-paratha' or 'dupli' are used in western and eastern parts of India [5]. Gay and bisexual identities are more common among same-sex or both-sex attracted males, respectively, from educated and middle or upper socioeconomic class.

A significant proportion of same-sex attracted men in India, irrespective of their sexual identities, are married or eventually get married due to societal expectations and family pressure, as getting married is seen as a duty to one's family and to sustain one's lineage $[6,7]$. For example, the first Integrated Behavioral and Biological Assessment (IBBA) study documented that about onefourth of the MSM participants had ever been married to women (16-37\%)-with $11.2 \%$ of kothis, $25 \%$ of double-deckers, $20 \%$ of panthis, and $61.2 \%$ of bisexual-identified MSM reported having ever been married [8]. Thus, bisexual behavior is not seen only among men who identify as 'bisexual', but also among MSM with diverse self-identities. Despite this awareness of the extent of bisexual behavior among even self-identified MSM, in general, HIV prevention interventions among MSM in India have remained predominantly focused on sexual risk reduction with male partners, with very limited attention to risk behaviors with female partners. Only recently the national HIV program explicitly acknowledged the need to address bisexual behavior among self-identified MSM and to promote safer sex with female partners of MSM as well [9]. Very few HIV programs focus on female partners of MSM in India. For instance, the Humsafar Trust, a community-based agency working with MSM in Mumbai, refers the female partners of its MSM clients to Family Planning Association of India (FPAI), where female partners are provided voluntary counselling and testing for sexually transmitted infections and HIV.
A recent meta-analysis on studies among MSMW in USA found that MSMW have relatively lower HIV prevalence when compared with MSMO, and less likely to engage in unprotected anal sex [10]. In India, there is limited and conflicting data on the possible differences, if any, on sexual risk behaviors and other factors associated with the prevalence of HIV and other sexually transmitted infections (STIs) among MSMW and MSMO. This means little understanding about whether and in what ways HIV prevention interventions need to be tailored depending on whether a person is MSMO or MSMW. One study from Bengaluru city in India that explicitly examined differences between MSMW and MSMO reported that MSMW were less likely to practice unprotected anal sex when compared with MSMO [11]. However, it did not examine differences in STI/HIV prevalence or intervention exposure.

Among the studies that reported HIV prevalence among married and unmarried MSM, one clinic-based study from Mumbai [12] and a multi-site community-based study [13] reported that married MSM were more likely than unmarried MSM to be HIV-positive, but another clinic-based study from Mumbai [14] could not find a statistically significant difference in HIV prevalence between married and single MSM.

To address these gaps in information and in order to inform designing HIV interventions tailored to MSMW's prevention needs, the aim of this paper is to compare and contrast HIV-related sexual risk behaviors, prevalence of HIV/STIs and HIV intervention exposure among MSMW and MSMO.

\section{Methods}

\section{Data Sources}

Data from a cross sectional bio-behavioral survey conducted among MSM in 2009/2010 were used for this analysis. The survey was conducted in 10 districts of three southern states of India-Andhra Pradesh, Maharashtra and Tamil Nadu. The inclusion criteria slightly differed in these states. In Andhra Pradesh and Maharashtra, the inclusion criterion was 'males aged 18 years and above who had any type of sex (oral, anal, or manual) with other males in the past one month', whereas in Tamil Nadu the inclusion criterion was 'males aged 18 years and above who had anal sex with other males in the past month in exchange for cash or in kind'. Following a rigorous sampling frame development, MSM were randomly sampled using time-location cluster sampling from cruising sites such as bus stands, cinema halls, parks, public toilets and other public places where MSM meet their potential male sexual partners. Two-stage sampling method was employed: at first stage, 
time-location clusters were selected using probability proportional to size (PPS); and in the second stage, respondents were recruited randomly from the selected clusters [15]. Written informed consent was obtained, following which interviews were conducted in private setting and blood and urine samples were collected. Ethical approvals were provided by Protection of Human Subjects Committee (PHSC) of FHI 360, Health Ministry Screening Committee (ICMR) and local ethical committees of the study implementing partners. Further details on the study methodology have been published elsewhere [16].

\section{Measures}

\section{Number and Types of Partners}

Data were collected on the number and types of partners. Types of partners included: regular partner or main partner-a person with whom the respondent feels committed, such as spouse, lover or boyfriend/girlfriend; paying partner-a person who have paid the respondent cash or kind in exchange for sex; casual partner-a stranger, friend or acquaintance with whom the respondent had sex and but not considered as a regular partner; and paid partner-a person with whom the respondent had paid money to have sex.

\section{$M S M W$ and MSMO}

As mentioned earlier, two categories of MSM were created for this analysis: (a) MSMO: those who reported having male regular partner, those who had a male paying partner in the past week, those who had any male paid partner in past one month and those who reported having any male casual partner (such as lover), and not reporting sex with any female partner in the past month; (b) MSMW: included those who had any of these above male partners and those who also reported having a female regular partner (such as wife or lover) and female paid partner in the past month. Since timeframe for the different partners (male and female) were current or within the past month, it was considered to be concurrent partners and hereafter will be referred to as such.

\section{Condom Use}

Condom use with different types of male and female partners was considered as the main outcome variable indicative of sexual risk. The survey included questions on condom use at last sex and consistency of condom use in the recent past (no specific timeframe was provided) with each type (regular, casual, paid, and paying) of male or female partners. Last sex condom use was examined separately for each partner type among MSMO and MSMW.

The main dependent variable was self-reported inconsistent condom use with any one of the four types of partners-separately examined for male and female partners. For each type of partner, participants who reported using condoms every time (during anal or vaginal sex) were considered to be consistent condom users, and those who reported using condoms most of the time, sometimes, and never were considered as inconsistent condom users. 'Inconsistent condom use with any type of male partners' was defined as having reported inconsistent condom use with any one of the four types (regular, casual, paying or paid) of male partners. Similarly 'inconsistent condom use with any type of female partners' was defined as having reported inconsistent condom use with any one of the two types (regular or paid) of female partners.

\section{HIV and STIS}

Blood samples were tested for HIV infection with a twotest algorithm using an enzyme immunoassay (J. Mitra, New Delhi) [16]. Blood samples were also tested for syphilis using rapid plasma reagin (RPR) and a confirmatory Treponema pallidum hemagglutination assay (TPHA). Positive RPR confirmation by TPHA was used to define reactive syphilis or lifetime syphilis. RPR titres of $\geq 1: 8$ with a confirmatory TPHA were defined as active or hightitre syphilis. Urine was tested using nucleic-acid amplification (Gen-Probe APTIMA COMBO 2) to assess $\mathrm{Ch}$ lamydia (CT) and gonococcal (GC) urethral infections [16]. For the analysis, a composite variable 'any STI' was defined as those testing positive for either syphilis, NG or CT.

\section{Socio-demographic and HIV Intervention Exposure Variables}

These include age, literacy status (reading and writing), years of education, marital status, occupation and sexual self-identification. The latter included: kothis-predominantly receptive partner during penetrative sex with men; double-deckers (DD) - both insertive and receptive; and panthis-predominantly insertive [17, 18]. Alcohol use was also asked, with frequent alcohol use defined as those who consumed alcohol every day or at least once a week [19]. Other measures included were: HIV self-risk perception (yes or no) and having ever taken an HIV test and collected the result (yes or no). Exposures to HIV intervention services were examined: contacted by peer educators for HIV information, received condoms from HIV intervention staff, and visited program STI clinic in the past year. In addition, membership in community-based 
organization of MSM (a formal group comprised of and managed by MSM) was also included.

\section{Data Analysis}

Data from all districts of the three states were merged for analysis. All estimates presented are not weighted. Bivariate and multivariable analyses were conducted using Stata (version 11.0). Chi square test was used to assess the associations between the independent variables and condom use outcome measures described above, with each of the partner type. Logistic regression was also conducted to identify differences, between MSMW and MSMO in terms of sexual risk behaviors (consistent condom use; condom use in last sex) and HIV/STI prevalence. Multivariable logistic regression was conducted: (a) to assess correlates of MSMW; and (b) to assess the sexual risk among MSMW and MSMO (for inconsistent condom use with any type of partners - separately for male and female partners). Odds ratios and $95 \%$ confidence intervals $(\mathrm{CI})$ were calculated after controlling for background variables in each model. Associations were considered significant for $p$ value less than 0.05 .

\section{Results}

\section{Sample Characteristics}

Among the total sample of 3739 MSM, about one-third (35\%,n=1343) were classified as MSMW and remaining $(65 \%, n=2396)$ as MSMO. Three-fourths $(75 \%)$ of MSMO self-identified as kothi, whereas nearly half (46\%) of MSMW identified as bisexual $(p<0.01)$. When compared with MSMW, significantly higher proportions of MSMO were young (18-25 years), completed secondary education, had never been married and reported sexual debut before 16 years of age. Almost equal proportion of MSMW and MSMO (40 and $38 \%$, respectively) reported frequent use of alcohol (every day or once a week) (Table 1).

\section{HIV-Related Risk Perception, Exposure to HIV Intervention and STI/HIV Prevalence}

\section{Overview of MSMW}

Although a majority of MSMW did not perceive to be at risk of HIV, in the past year, $75 \%$ had tested for HIV and returned to collect test results. About two-thirds (70\%) of MSMW had received services for HIV prevention (peer education, free condoms, and STI clinic check-ups) and $50 \%$ reported being members of community-based organizations. Thirteen percent tested positive for HIV and $3.5 \%$ had evidence of active syphilis.

\section{Comparison of MSMW to MSMO}

A relatively lower proportion of MSMW perceived themselves at risk for HIV (21 vs. $27 \%, p<0.001$ ), and had ever been tested for HIV and collected test results (75 vs. $81 \%, p<0.001)$ compared to MSMO. Exposures to any type of HIV intervention services as well as CBO membership were significantly lower among MSMW. No significant differences were observed in HIV or STI prevalence among two groups (Table 1).

\section{Partner Characteristics and Sexual Behaviors}

\section{Overview of MSMW}

Over $75 \%$ of MSMW were married and living with a female partner. However, only $4 \%$ of MSMW reported that their female regular partners were aware of their same-sex sexual behavior. MSMW reported having all types of male partners concurrently within the past month in the following order: male casual partners $(77 \%)$, male regular partners $(55 \%)$ and paying partners $(47 \%)$. A vast majority $(93 \%)$ reported having a current female regular partner and $14.6 \%$ reported having sex with a female sex worker in the past month (Table 2).

The percentage of condom use during last sexual act reported by MSMW was high with all types of male partners: with male regular partners it was $89 \%$ and with other male partners it was over $90 \%$. Consistent condom use among MSMW was higher among casual and paid male partners (over $80 \%$ ) compared with male regular $(72.4 \%)$ and paying partners $(70.8 \%)$.

Last sex condom use was higher with female paid partners (93\%) (23\%). Similarly, consistent condom use was only $14.6 \%$ with female regular partners, but higher with female paid partners $(83 \%)$.

\section{Comparison of MSMW to MSMO}

About $3 \%$ of MSMO reported being currently married. Similar to MSMW, MSMO reported having all types of male partners. However, MSMO had higher proportion of male regular partners $(74 \%)$ and male paying partners (73\%), but fewer male paid and male casual partners. There was no statistically significant difference between 
Table 1 Profile of men have sex with both men and women (MSMW) and men who have sex with men only (MSMO), IBBA-2, 2009/2010

\begin{tabular}{|c|c|c|c|}
\hline Variables & "MSMW" $(n=1343) \%(n)$ & "MSMO" $(n=2396) \%(n)$ & $p$ value \\
\hline \multicolumn{4}{|l|}{ Socio-demographics } \\
\hline \multicolumn{4}{|l|}{ Self-identity } \\
\hline Kothi & $23.3(314)$ & $74.5(1785)$ & \multirow[t]{4}{*}{0.000} \\
\hline Panthi & $15.7(211)$ & $12.4(298)$ & \\
\hline Double-decker & 14.7 (198) & $10.1(243)$ & \\
\hline Bisexual & $46.1(620)$ & $2.9(70)$ & \\
\hline \multicolumn{4}{|l|}{ Age group (years) } \\
\hline $18-25$ & $29.4(395)$ & $57.1(1369)$ & \multirow[t]{3}{*}{0.000} \\
\hline $26-35$ & $47.3(636)$ & $34.3(825)$ & \\
\hline 36 and older & $23.2(312)$ & $8.5(204)$ & \\
\hline \multicolumn{4}{|l|}{ Education } \\
\hline Illiterate & $18.0(243)$ & $9.6(230)$ & \multirow[t]{3}{*}{0.000} \\
\hline Up to secondary education & $32.3(435)$ & $27.5(661)$ & \\
\hline Above secondary education & $49.5(665)$ & $62.8(1505)$ & \\
\hline \multicolumn{4}{|l|}{ Current marital status } \\
\hline Never married & $24.0(323)$ & 95.9 (2298) & \multirow[t]{3}{*}{0.000} \\
\hline Currently married & $75.4(1013)$ & $2.6(64)$ & \\
\hline Divorced/widowed/separated & $0.52(7)$ & $1.4(34)$ & \\
\hline \multicolumn{4}{|l|}{ Occupation } \\
\hline Unemployed/students & $7.1(96)$ & $14.9(357)$ & \multirow[t]{5}{*}{0.000} \\
\hline Manual laborers & $31.9(429)$ & $28.5(683)$ & \\
\hline Business/Govt. or Pvt. employee/professional & $58.3(783)$ & $49.7(1192)$ & \\
\hline Masseur/sex worker & $1.7(23)$ & $6.1(148)$ & \\
\hline Transport workers & $0.8(12)$ & $0.6(16)$ & \\
\hline \multicolumn{4}{|l|}{ Age at sexual debut with a male } \\
\hline Less than 16 & $23.4(315)$ & $44.0(1055)$ & \multirow[t]{2}{*}{0.000} \\
\hline 16 and above & $76.5(1028)$ & $55.9(1341)$ & \\
\hline \multicolumn{4}{|l|}{ Age at sexual debut with a female } \\
\hline Less than 16 & $2.2(30)$ & $6.2(19)$ & \multirow[t]{2}{*}{0.000} \\
\hline 16 and above & $97.7(1280)$ & 93.7 (286) & \\
\hline \multicolumn{4}{|l|}{ Alcohol use } \\
\hline Everyday & $5.3(72)$ & $6.0(144)$ & \multirow[t]{5}{*}{0.002} \\
\hline Once a week & $35.0(471)$ & $32.0(767)$ & \\
\hline Less than once a week & $22.2(299)$ & $19.1(458)$ & \\
\hline Not in the past month & $6.8(92)$ & $6.4(154)$ & \\
\hline Never & $30.4(409)$ & $36.4(873)$ & \\
\hline \multicolumn{4}{|l|}{ Risk perception and HIV testing } \\
\hline \multicolumn{4}{|l|}{ Self-perceived HIV risk } \\
\hline No & $79.2(1064)$ & $73.2(1755)$ & \multirow[t]{2}{*}{0.000} \\
\hline Yes & 20.7 (279) & $26.7(641)$ & \\
\hline \multicolumn{4}{|c|}{ Ever been tested and returned to collect HIV test results } \\
\hline No & $24.9(335)$ & $18.6(446)$ & \multirow[t]{2}{*}{0.000} \\
\hline Yes & $75.0(1008)$ & $81.3(1950)$ & \\
\hline \multicolumn{4}{|c|}{ Use of HIV-related prevention services (in the past year) } \\
\hline Received peer education & & & \\
\hline No & $30.5(410)$ & $20.4(490)$ & 0.000 \\
\hline Yes & $69.4(933)$ & $79.5(1906)$ & \\
\hline
\end{tabular}


Table 1 continued

\begin{tabular}{|c|c|c|c|}
\hline Variables & "MSMW" $(n=1343) \%(n)$ & "MSMO" $(n=2396) \%(n)$ & $p$ value \\
\hline \multicolumn{4}{|c|}{ Received condoms from NGOs/CBOs } \\
\hline No & $31.0(417)$ & $20.3(488)$ & \multirow[t]{2}{*}{0.000} \\
\hline Yes & $68.9(926)$ & $79.6(1908)$ & \\
\hline \multicolumn{4}{|c|}{ Visited NGO-managed STI clinics } \\
\hline No & $34.2(460)$ & $25.8(620)$ & \multirow[t]{2}{*}{0.000} \\
\hline Yes & $65.7(883)$ & $74.1(1776)$ & \\
\hline \multicolumn{4}{|c|}{ Composite indicator: exposure to HIV intervention } \\
\hline No & $29.5(397)$ & $19.4(465)$ & \multirow[t]{2}{*}{0.000} \\
\hline Yes & $70.4(946)$ & $80.5(1931)$ & \\
\hline \multicolumn{4}{|c|}{ Membership in a $\mathrm{CBO}$} \\
\hline No & $50.3(676)$ & $46.5(1115)$ & \multirow[t]{2}{*}{0.026} \\
\hline Yes & $49.6(667)$ & $53.4(1281)$ & \\
\hline \multicolumn{4}{|c|}{ HIV and STIs (lab tests) } \\
\hline \multicolumn{4}{|l|}{ HIV } \\
\hline Negative & $86.9(1167)$ & $87.7(2103)$ & \multirow[t]{2}{*}{0.438} \\
\hline Positive & $13.1(176)$ & $12.2(293)$ & \\
\hline \multicolumn{4}{|l|}{ Syphilis } \\
\hline Negative & $92.9(1248)$ & $94.4(2263)$ & \multirow[t]{2}{*}{0.062} \\
\hline Positive & $7.0(95)$ & $5.5(133)$ & \\
\hline \multicolumn{4}{|c|}{ High-titre syphilis } \\
\hline Negative & 96.5 (1296) & $96.8(2320)$ & \multirow[t]{2}{*}{0.590} \\
\hline Positive & $3.5(47)$ & $3.1(76)$ & \\
\hline \multicolumn{4}{|c|}{ Any STI (excluding HIV) } \\
\hline Negative & $92.2(1239)$ & $93.6(2243)$ & \multirow[t]{2}{*}{0.115} \\
\hline Positive & 7.7 (104) & $6.3(153)$ & \\
\hline
\end{tabular}

NGO/CBO Non-governmental Organization/Community-Based Organization

MSMW and MSMO in condom use during last anal sex with any type of male partner (Table 2).

However, when compared with MSMW, consistent condom use was lower among all types of male partners of MSMO. With significantly lower proportion of MSMO reporting consistent condom use with male regular $(65.8$ vs. $72.4 \%, p<0.01)$ and with male casual $(77.7$ vs. $83.1 \%, p<0.01$ ) partners (Tables 3, 4).

When compared with MSMW, higher proportion of MSMO (35.2 vs. $27 \%, p<0.001)$ reported inconsistent condom use with any type of male partners. Similarly, use of exclusive water-based lubricants was higher among MSMO (39 vs. $26 \%, p<0.001)$.

\section{Findings from Multivariate Analysis}

\section{Overview of MSMW}

Multivariate analysis identified significant correlates of MSMW group. MSMW were less likely to identify as kothi [AOR $0.07(0.05-0.09)$ ] and more likely to be $>26$ years of age [AOR 4.49 (3.69-5.47)]. MSMW were less likely to have a male regular partner [AOR 0.73 (0.60-0.87)] compared to MSMO; however, there was no significant differences from MSMO in having other types of male partners. MSMW were less likely to be inconsistent condom users with any type of male partners [AOR 0.73 (0.60-0.88)] compared with MSMO. While there was no difference in self-perception of HIV risk or exposure to services such as peer education and condoms, MSMW were more likely to have received STI clinical services [AOR 1.60 (1.10-2.32)] compared to MSMO.

\section{Associations with Inconsistent Condom Use: Comparison of MSMW and MSMO}

\section{Inconsistent Condom Use with Male Partners}

Correlates of inconsistent condom use with any type of male partners was examined separately for MSMW and MSMO. The common factors that were found significantly 
Table 2 Partners-related characteristics and sexual risk behaviors of MSMW and MSMO

\begin{tabular}{|c|c|c|c|}
\hline Variables & "MSMW" $(n=1343) \%(n)$ & "MSMO" $(n=2396) \%(n)$ & $p$ value \\
\hline \multicolumn{4}{|c|}{ Types of partners ${ }^{\mathrm{a}}$} \\
\hline \multicolumn{4}{|c|}{ Male regular partners } \\
\hline No & $44.2(594)$ & $25.7(616)$ & \multirow[t]{2}{*}{0.000} \\
\hline Yes & $55.7(749)$ & $74.2(1778)$ & \\
\hline \multicolumn{4}{|c|}{ Male paying partners } \\
\hline No & $52.9(711)$ & $27.3(656)$ & \multirow[t]{2}{*}{0.000} \\
\hline Yes & $47.0(632)$ & $72.6(1740)$ & \\
\hline \multicolumn{4}{|c|}{ Male paid partners } \\
\hline No & 80.9 (1086) & $86.6(2074)$ & \multirow[t]{2}{*}{0.000} \\
\hline Yes & $19.0(256)$ & $13.3(319)$ & \\
\hline \multicolumn{4}{|c|}{ Male casual partners } \\
\hline No & $22.8(307)$ & $29.6(709)$ & \multirow[t]{2}{*}{0.000} \\
\hline Yes & $77.1(1035)$ & $70.4(1686)$ & \\
\hline \multicolumn{4}{|c|}{ Female regular partners } \\
\hline No & $6.2(84)$ & - & \multirow[t]{2}{*}{0.000} \\
\hline Yes & $93.7(1259)$ & - & \\
\hline \multicolumn{4}{|c|}{ Female paid partners } \\
\hline No & $85.3(1146)$ & - & \multirow[t]{2}{*}{0.000} \\
\hline Yes & $14.6(197)$ & - & \\
\hline \multicolumn{4}{|c|}{ Characteristics of female regular and paid partners of MSMW } \\
\hline \multicolumn{4}{|c|}{ Currently married } \\
\hline No & $24.5(330)$ & - & - \\
\hline Yes & $75.4(1013)$ & - & - \\
\hline \multicolumn{4}{|c|}{ Regular female partner } \\
\hline \multicolumn{4}{|l|}{ Living together } \\
\hline No & $23.2(312)$ & - & - \\
\hline Yes & $76.7(1031)$ & - & - \\
\hline \multicolumn{4}{|c|}{ Female regular partner's awareness of husband's same-sex sexual behavior } \\
\hline No & $96.2(1292)$ & - & - \\
\hline Yes & $3.8(51)$ & - & - \\
\hline \multicolumn{4}{|c|}{ Number of sex acts with female regular partner in the past month } \\
\hline$\leq 7$ & $52.7(617)$ & - & - \\
\hline $8-14$ & $16.3(191)$ & - & - \\
\hline 15 and above & $30.8(361)$ & - & - \\
\hline \multicolumn{4}{|l|}{ Paid partner } \\
\hline \multicolumn{4}{|c|}{ Number of times bought sex with females in the past one month } \\
\hline$\leq 4$ & $82.6(148)$ & - & - \\
\hline 5 and above & $17.3(31)$ & - & - \\
\hline \multicolumn{4}{|c|}{ Condom use behaviors } \\
\hline \multicolumn{4}{|c|}{ Last time condom use (anal or vaginal sex) } \\
\hline With male regu & & & \\
\hline No & $10.6(76)$ & $13.3(229)$ & 0.071 \\
\hline Yes & $89.3(637)$ & $86.6(1490)$ & \\
\hline With male pay & & & \\
\hline No & $2.2(14)$ & $1.8(30)$ & 0.455 \\
\hline Yes & 97.7 (598) & $98.2(1635)$ & \\
\hline With male paid & & & \\
\hline No & $6.3(16)$ & 6.0 (19) & 0.910 \\
\hline
\end{tabular}


Table 2 continued

\begin{tabular}{|c|c|c|c|}
\hline Variables & "MSMW" $(n=1343) \%(n)$ & "MSMO" $(n=2396) \%(n)$ & $p$ value \\
\hline Yes & $93.7(238)$ & $93.9(294))$ & \\
\hline \multicolumn{4}{|l|}{ With male casual partner } \\
\hline No & $2.0(20)$ & $1.6(26)$ & \multirow{2}{*}{0.466} \\
\hline Yes & $97.9(944)$ & $98.3(1527)$ & \\
\hline \multicolumn{4}{|l|}{ With female regular partner } \\
\hline No & $76.4(955)$ & - & - \\
\hline Yes & $23.5(294)$ & - & - \\
\hline \multicolumn{4}{|l|}{ With female paid partner } \\
\hline No & $4.5(9)$ & - & - \\
\hline Yes & $95.4(188)$ & - & - \\
\hline \multicolumn{4}{|l|}{ Consistent condom use (in general) } \\
\hline \multicolumn{4}{|l|}{ With regular male partners } \\
\hline No & $27.5(187)$ & $34.2(564)$ & \multirow[t]{2}{*}{0.002} \\
\hline Yes & $72.4(492)$ & $65.8(1085)$ & \\
\hline \multicolumn{4}{|l|}{ With male paying partners } \\
\hline No & $29.1(178)$ & $31.4(525)$ & \multirow[t]{2}{*}{0.284} \\
\hline Yes & $70.8(433)$ & $68.5(1143)$ & \\
\hline \multicolumn{4}{|l|}{ With male paid partners } \\
\hline No & $18.6(47)$ & $22.9(71)$ & \multirow[t]{2}{*}{0.211} \\
\hline Yes & $81.3(205)$ & $77.0(238)$ & \\
\hline \multicolumn{4}{|l|}{ With casual male partners } \\
\hline No & $16.8(163)$ & $22.2(349)$ & \multirow[t]{2}{*}{0.001} \\
\hline Yes & $83.1(804)$ & $77.7(1222)$ & \\
\hline \multicolumn{4}{|l|}{ With regular female partners } \\
\hline No & $85.3(1067)$ & - & - \\
\hline Yes & $14.6(183)$ & - & - \\
\hline \multicolumn{4}{|l|}{ With paid female partners } \\
\hline No & $17.2(34)$ & - & - \\
\hline Yes & $82.7(163)$ & - & - \\
\hline \multicolumn{4}{|l|}{ Inconsistency in condom use with } \\
\hline Any type of male partners (regular, paying, paid, or casual) & $27.0(363)$ & $35.2(844)$ & 0.000 \\
\hline Female partners (paid or regular) & $77.0(1035)$ & - & 0.000 \\
\hline Both male and female partners & $23.8(320)$ & - & 0.000 \\
\hline \multicolumn{4}{|l|}{ Lubricant use $^{\mathrm{a}}$} \\
\hline Non-users/mixed users & $74.2(995)$ & $60.7(1453)$ & 0.000 \\
\hline Exclusive use of water-based lubricants & $25.8(346)$ & $39.2(937)$ & \\
\hline
\end{tabular}

Total and \% may not add up due to missing values

${ }^{a}$ Mixed users include those who reported using both water and oil based lubricants

associated with inconsistent condom use with any type of male partners were: age $>26$ years [MSMW: AOR 1.62 (1.07-2.46); MSMO: AOR 1.58 (1.32-1.90)] and frequent alcohol use [MSMW: AOR 1.67 (1.28-2.19); MSMO: AOR 1.48 (1.24-1.78)].

\section{Significant Unique Correlates in MSMW Group}

Among MSMW, with kothis as the reference group, inconsistent condom use with any type of male partners was less likely among panthis [AOR $0.51(0.30-0.85)]$ and bisexual-identified MSM [AOR 0.40 (0.28-0.56)]. Among MSMO, with kothis as the reference group, inconsistent condom use with any type of male partners was significantly lower among all other subgroups [panthis $=$ AOR $0.35 \quad(0.25-0.50) ; \quad$ bisexuals $=$ AOR 0.36 (0.20-0.64); DDs = AOR 0.35 (0.20-0.65)].

Among MSMW, those who were exposed to STI clinical services were less likely to be inconsistent condom users with any type of male partners [AOR $0.57(0.21-0.66)]$. 
Table 3 Multivariate associations of being men who have sex with men and women-MSMW (vs. MSMO), IBBA-2, 2009/2010 ( $n=1343$ )

\begin{tabular}{|c|c|c|}
\hline Variables & $\begin{array}{l}\text { MSMW } \\
\text { Adjusted odds ratio (95\% confidence interval) }\end{array}$ & $p$ value \\
\hline \multicolumn{3}{|l|}{ Self-identity } \\
\hline Non-kothi ${ }^{\mathrm{a}}$ & Referent & \\
\hline Kothi & $0.07(0.06-0.09)$ & 0.000 \\
\hline \multicolumn{3}{|l|}{ Age (years) } \\
\hline$\leq 25$ & Referent & \\
\hline 26 and older & $4.45(3.66-5.42)$ & 0.000 \\
\hline \multicolumn{3}{|l|}{ Education } \\
\hline Illiterate & Referent & \\
\hline Literate & $0.44(0.35-0.55)$ & 0.000 \\
\hline \multicolumn{3}{|l|}{ Occupation } \\
\hline Organized sector (Govt./Private), unemployed/students & Referent & \\
\hline Manual laborers & $2.18(1.64-2.91)$ & 0.000 \\
\hline \multicolumn{3}{|l|}{ Alcohol use } \\
\hline No & Referent & \\
\hline Yes & $1.11(0.94-1.33)$ & 0.218 \\
\hline \multicolumn{3}{|l|}{ Having male regular partner } \\
\hline No & Referent & \\
\hline Yes & $0.75(0.62-0.89)$ & 0.001 \\
\hline \multicolumn{3}{|l|}{ Having male paying partners } \\
\hline No & Referent & \\
\hline Yes & $0.91(0.75-1.12)$ & 0.400 \\
\hline \multicolumn{3}{|l|}{ Having male casual partners } \\
\hline No & Referent & \\
\hline Yes & $0.94(0.77-1.15)$ & 0.532 \\
\hline \multicolumn{3}{|c|}{ Inconsistent condom use with any type of male partners (regular, paying, paid, casual) } \\
\hline No & Referent & \\
\hline Yes & $0.82(0.67-0.98)$ & 0.036 \\
\hline \multicolumn{3}{|l|}{ Composite indicator: exposure to HIV intervention } \\
\hline No & Referent & \\
\hline Yes & $1.19(0.96-1.47)$ & 0.104 \\
\hline \multicolumn{3}{|l|}{ Self-perceived HIV risk } \\
\hline No & Referent & \\
\hline Yes & $0.93(0.76-1.14)$ & 0.473 \\
\hline \multicolumn{3}{|l|}{ Membership in a CBO } \\
\hline No & Referent & \\
\hline Yes & $0.99(0.99-1.00)$ & 0.909 \\
\hline \multicolumn{3}{|l|}{ Exclusive use of water-based lubricants } \\
\hline No & Referent & \\
\hline Yes & $0.79(0.66-0.96)$ & 0.019 \\
\hline \multicolumn{3}{|l|}{ Syphilis } \\
\hline Negative & Referent & \\
\hline Positive & $1.10(0.78-1.55)$ & 0.577 \\
\hline
\end{tabular}

a 'Non-kothi' includes MSM who identified as panthis, double-deckers, or bisexuals

Model $\chi^{2}(16)=1439.45, p<0.001$, log likelihood $=-1711.78$, pseudo $R^{2}=0.296$ 
Table 4 Multivariate associations of inconsistent condom use with any type (regular, casual, paying or paid) of male partners and female partners (regular or paid) among MSMW and MSMO, 2009/2010

\begin{tabular}{|c|c|c|c|c|c|c|}
\hline \multirow[t]{3}{*}{ Variables } & \multicolumn{4}{|l|}{ MSMW } & \multicolumn{2}{|l|}{ MSMO } \\
\hline & \multicolumn{2}{|c|}{$\begin{array}{l}\text { Inconsistent condom use with any } \\
\text { type of male partners }{ }^{\mathrm{a}}\end{array}$} & \multicolumn{2}{|c|}{$\begin{array}{l}\text { Inconsistent condom use with any } \\
\text { type of female partners }{ }^{b}\end{array}$} & \multicolumn{2}{|c|}{$\begin{array}{l}\text { Inconsistent condom use with any } \\
\text { type of male partners }{ }^{\mathrm{c}}\end{array}$} \\
\hline & $\begin{array}{l}\text { Adjusted odds ratio } \\
(95 \% \mathrm{CI})\end{array}$ & $p$ value & $\begin{array}{l}\text { Adjusted odds ratio } \\
(95 \% \mathrm{CI})\end{array}$ & $p$ value & $\begin{array}{l}\text { Adjusted odds ratio } \\
(95 \% \mathrm{CI})\end{array}$ & $p$ value \\
\hline \multicolumn{7}{|l|}{ Self-identity } \\
\hline Kothi & Referent & & Referent & & Referent & \\
\hline Panthi & $0.51(0.31-0.85)$ & 0.009 & $0.15(0.07-0.31)$ & 0.000 & $0.35(0.25-0.50)$ & 0.000 \\
\hline Double-decker & $0.87(0.59-1.28)$ & 0.487 & $0.36(0.18-0.75)$ & 0.006 & $0.71(0.53-0.96)$ & 0.028 \\
\hline Bisexual & $0.40(0.28-0.56)$ & 0.000 & $0.29(0.16-0.54)$ & 0.000 & $0.36(0.20-0.64)$ & 0.001 \\
\hline \multicolumn{7}{|l|}{ Age (years) } \\
\hline$\leq 25$ & Referent & & Referent & & Referent & \\
\hline 26 or older & $1.62(1.07-2.46)$ & 0.022 & $6.8(4.84-9.66)$ & 0.000 & $1.59(1.32-1.91)$ & 0.000 \\
\hline \multicolumn{7}{|l|}{ Education } \\
\hline Illiterate & Referent & & Referent & & Referent & \\
\hline Literate & $1.02(0.75-1.38)$ & 0.885 & $0.42(0.262-0.66)$ & 0.000 & $1.02(0.77-1.34)$ & 0.863 \\
\hline \multicolumn{7}{|l|}{ Currently married } \\
\hline No & Referent & & - & & - & \\
\hline Yes & $0.79(0.52-1.18)$ & 0.253 & - & - & - & - \\
\hline \multicolumn{7}{|l|}{ Occupation } \\
\hline $\begin{array}{l}\text { Organized sector/unemployed/ } \\
\text { students }\end{array}$ & Referent & & Referent & & Referent & \\
\hline Manual laborers & $1.42(0.78-2.56)$ & 0.240 & $1.54(0.91-2.61)$ & 0.108 & $1.04(0.80-1.37)$ & 0.729 \\
\hline \multicolumn{7}{|l|}{ Alcohol use } \\
\hline No & Referent & & Referent & & Referent & \\
\hline Yes & $1.67(1.27-2.19)$ & 0.000 & $0.67(0.48-0.93)$ & 0.017 & $1.48(1.24-1.78)$ & 0.000 \\
\hline \multicolumn{7}{|l|}{ Received peer education } \\
\hline No & Referent & & Referent & & Referent & \\
\hline Yes & $1.98(0.77-5.07)$ & 0.155 & $1.52(0.55-4.18)$ & 0.417 & $0.64(0.30-1.36)$ & 0.252 \\
\hline \multicolumn{7}{|c|}{ Received condoms from NGOs/CBOs } \\
\hline No & Referent & & Referent & & Referent & \\
\hline Yes & $0.93(0.37-2.29)$ & 0.870 & $1.00(0.39-2.50)$ & 0.983 & $0.93(0.44-1.36)$ & 0.245 \\
\hline \multicolumn{7}{|l|}{ Visited NGO-managed STI clinics } \\
\hline No & Referent & & Referent & & Referent & \\
\hline Yes & $0.57(0.21-0.66)$ & 0.001 & $1.10(0.54-2.29)$ & 0.780 & $0.77(0.54-1.09)$ & 0.151 \\
\hline \multicolumn{7}{|l|}{ Self-perceived HIV risk } \\
\hline No & Referent & & Referent & & Referent & \\
\hline Yes & $1.58(1.17-2.13)$ & 0.002 & $0.77(0.51-1.14)$ & 0.196 & $1.14(0.94-1.38)$ & 0.198 \\
\hline \multicolumn{7}{|c|}{ Female regular partner's awareness of husband's same-sex sexual behavior } \\
\hline No & NA & & Referent & & NA & - \\
\hline Yes & & & $0.36(0.16-0.81)$ & 0.015 & - & \\
\hline \multicolumn{7}{|l|}{ Membership in a $\mathrm{CBO}$} \\
\hline No & Referent & & Referent & & Referent & \\
\hline Yes & $0.99(0.98-1.00)$ & 0.588 & $1.00(0.99-1.01)$ & 0.324 & $0.99(0.98-1.00)$ & 0.381 \\
\hline \multicolumn{7}{|c|}{ Exclusive use of water-based lubricants } \\
\hline No & Referent & & - & & Referent & \\
\hline Yes & $1.05(0.78-1.42)$ & 0.736 & - & - & $1.08(0.91-1.31)$ & 0.364 \\
\hline \multicolumn{7}{|l|}{ Syphilis } \\
\hline No & Referent & & Referent & & Referent & \\
\hline
\end{tabular}


Table 4 continued

\begin{tabular}{|c|c|c|c|c|c|c|}
\hline \multirow[t]{3}{*}{ Variables } & \multicolumn{4}{|l|}{ MSMW } & \multicolumn{2}{|l|}{ MSMO } \\
\hline & \multicolumn{2}{|c|}{$\begin{array}{l}\text { Inconsistent condom use with any } \\
\text { type of male partners }{ }^{\mathrm{a}}\end{array}$} & \multicolumn{2}{|c|}{$\begin{array}{l}\text { Inconsistent condom use with any } \\
\text { type of female partners }\end{array}$} & \multicolumn{2}{|c|}{$\begin{array}{l}\text { Inconsistent condom use with any } \\
\text { type of male partners }{ }^{c}\end{array}$} \\
\hline & $\begin{array}{l}\text { Adjusted odds ratio } \\
(95 \% \mathrm{CI})\end{array}$ & $p$ value & $\begin{array}{l}\text { Adjusted odds ratio } \\
(95 \% \mathrm{CI})\end{array}$ & $p$ value & $\begin{array}{l}\text { Adjusted odds ratio } \\
(95 \% \mathrm{CI})\end{array}$ & $p$ value \\
\hline Yes & $0.96(0.58-1.58)$ & 0.859 & $0.88(0.45-1.76)$ & 0.736 & $1.58(1.07-2.32)$ & 0.021 \\
\hline \multicolumn{7}{|l|}{ HIV } \\
\hline Negative & Referent & & Referent & & Referent & \\
\hline Positive & $0.81(0.55-1.19)$ & 0.285 & $1.42(0.81-2.50)$ & 0.219 & $0.75(0.56-0.99)$ & 0.043 \\
\hline \multicolumn{7}{|c|}{ Inconsistent condom use with any type of male partners } \\
\hline No & NA & & Referent & & - & \\
\hline Yes & - & - & $7.45(4.9-12.34)$ & 0.000 & - & - \\
\hline
\end{tabular}

${ }^{\text {a }}$ Prob $>\chi^{2}=0.000$, log likelihood $=-88.5$, pseudo $R^{2}=0.057$

b Prob $>\chi^{2}=0.000$; log likelihood $=-445.52$, pseudo $R^{2}=0.308$

${ }^{\mathrm{c}}$ Prob $>\chi^{2}=0.000 ; \log$ likelihood $=-138.04$, pseudo $R^{2}=0.045$

Other two HIV intervention exposure variables (having received peer education or condoms) were not found to have statistically significant association with inconsistent condom use with both MSMW and MSMO groups. Presence of self-perceived HIV risk was found to be significantly associated with inconsistent condom use with any type of male partners among MSMW [AOR 1.44 (1.06-1.94)], but not with MSMO.

\section{Significant Unique Correlates in MSMO Group}

MSMO who were syphilis positive [AOR 1.58 (1.07-2.32)] were more likely to be inconsistent condom users; whereas, MSMO who were HIV positive [AOR $0.75(0.56-0.99)]$ were less likely to be inconsistent condom users.

\section{Inconsistent Condom Use with Female Partners}

Similar to the pattern observed in significant correlates of inconsistent condom use with male partners, MSMW who were 26 years and above [AOR 6.8 (4.84-9.66)] were more likely to be inconsistent condom users with any type of female partners. However, frequent alcohol users [AOR $0.42(0.26-0.66)]$ were less likely to be inconsistent condom users with their female partners.

Similar to the pattern with male partners, compared to kothi-identified MSMW, MSMW who identified as panthi [AOR $0.15(0.07-0.31)$ ], DD [AOR $0.36(0.18-0.75)]$ and bisexual [AOR $0.29(0.16-0.54)$ ] were significantly less likely to be inconsistent condom users with their female partners. Exposure to HIV intervention was not found to be associated with inconsistent condom use with female partners. MSMW who reported that their female partners were aware of their same-sex sexual behaviors [AOR 0.36
(0.16-0.81)] were found less likely to be inconsistent condom users with their female partners. MSMW who were inconsistent condom users with any type of male partners [AOR 7.45 (4.9-12.34)] were more likely to inconsistent condom users with any type of female partners as well.

\section{Discussion}

This analysis based on a large cross-sectional survey among MSM in southern India has found that, when compared with MSMO, in general, MSMW have relatively less HIV-related sexual risk behaviors with their male partners, even after controlling for HIV intervention exposure. Despite this lower sexual risk among MSMW, there were no significant differences in HIV or STI prevalence between MSMW and MSMO. We found evidence that MSMW concurrently have unprotected sex with both male and female partners, increasing the chances of HIV transmission risk to partners of both sexes and to themselves.

\section{Prevalence of Bisexual Behavior}

Our findings that about one-third of MSM engage in heterosexual behavior as well is consistent with findings from other published studies among MSM in India. For example, other studies conducted among community-based samples of MSM such as a study from Bengaluru [11] reported $30 \%$ had bisexual behavior, and the first round of IBBA reported that $15-45 \%$ of different subgroups of MSM had female regular partners [13]. 


\section{Differences in HIV and STI Prevalence Among MSMW and MSMO}

The present study found that MSMW and MSMO had high prevalence of HIV and syphilis, similar in magnitude to the high HIV and STI prevalence found in a community-based [13] and a clinic-based study among MSM [12]. But unlike these two studies which reported that married MSM were more likely to have HIV infection than single MSM (29 vs. $18 \%$ in Brahmam et al. [13] and 23 vs. $9 \%$ in Kumta et al. [12]), the present study did not find any significant differences in HIV or syphilis prevalence among MSMW and MSMO, after controlling for HIV intervention exposure. It is possible that MSMW at higher risk might not have been captured in this study as after marriage many MSM stop coming to community-based agencies or even to cruising sites to avoid being discriminated by unmarried MSM [20].

\section{Age and Identity Differences Between MSMW and MSMO}

We found that MSMW were more likely to be aged 26 years and above, and more likely to have non-kothi identities, consistent with the findings from another study that explicitly compared MSMW and MSMO in Bangalore city [11]. It has been previously documented that even same-sex attracted males in India eventually get married to woman as they see marriage as a duty to one's family in this primarily collectivistic culture where family occupies a central role and arranged marriage is still widely prevalent $[3,21]$. However, marital status was not a significant correlate of being MSMW, which means even in absence of marriage MSM in different subgroups have sex with females. The finding that MSMW are more likely to be nonkothi identities could be possibly explained by the relatively more fluid nature of sexual behaviors among other subgroups of MSM (panthis, double-deckers and bisexuals) when compared with kothis. However, it is also possible that in our sample, kothis might have concealed their bisexual behavior or their marital status because of the stigma within kothi communities in relation to bisexual behavior and marriage.

\section{Differences in Condom Use Between MSMW and MSMO}

Our study findings showed that, when compared with MSMO, a higher proportion of MSMW consistently used condoms. Similarly, MSMW were less likely to be inconsistent condom users than MSMO with their male partners. These findings are consistent with the Bangalore study [11] that compared MSMW and MSMO, as well as a study that examined bisexual concurrency among South African
MSM [22]. Our finding that MSMW, in general, have high rates of condom use than MSMO, could not be explained by exposure to HIV interventions, as it was not found to be significantly different between MSMW and MSMO.

Future qualitative studies can explore whether a sense of responsibility to prevent transmission of HIV to their wives and children motivates MSMW to be more consistent in condom use with their male partners. However, the proportions of MSMW with inconsistent condom use with their male partners of any type are still high enough to warrant further attention towards improving consistent condom use with their male partners.

\section{Condom Use with Female Partners}

Being aged 26 years and above was significantly associated with inconsistent condom use with female partners whereas frequent use of alcohol was significantly less likely to be associated with inconsistent condom use with female partners. Also, when compared with kothis, MSMW who self-identified as panthi, DD or bisexual were less likely to be inconsistent condom users with their female partners, consistent with another study conducted among MSM in Bangalore, South India [6]. While the association between frequent alcohol use and inconsistent condom use can be understood [19], it is not clear how the self-identities of MSMW might account for the differences in their condom use with their female partners, given the paucity of research in this area in India.

Our key finding from bivariate analyses that those MSMW who reported inconsistent condom use with any one of the four types of male partners are also likely to be inconsistent condom users with their female partners indicate that partners of either gender of MSMW are at-risk for HIV and STIs, and MSMW too can get infected by or infect partners of either gender. Bivariate analyses also indicated that MSMW are more likely to inconsistently use condoms with their female regular partners compared to other types of female partners. This is possibly because condom use, in general, with in marital relationships is seen as affecting intimacy and possible indication of extramarital sex [23]. Also, the widespread use of family planning technologies (oral contraceptives or tubectomy) by women in India may prevent married MSM from justifying the use of condoms as a contraceptive device [24].

Disclosure of one's sexuality to spouse is very rare in our sample, as confirmed in other Indian studies [25, 26] and a South African study [22]. It is not clear whether disclosure of one's sexuality can facilitate safer sex practices with their spouse, even though multivariate analysis in this study showed that those MSMW whose spouse were aware of their husband's sexuality were more likely to report consistent condom use with their female partners. 
The connection between disclosure of one's sexuality to spouse and other female partners, and condom use needs to be further examined through qualitative or mixed methods studies.

\section{Limitations}

This current analysis has several limitations. The samples were from MSM accessing cruising sites in Western and Southern Indian states with a long history of HIV interventions among MSM. This issue, along with a minor difference in the inclusion criterion used in one of the three study states (Tamil Nadu), preclude generalizing the findings to even to MSM accessing cruising sites in India. Another limitation could be in relation to how we operationalized the definitions of MSMO and MSMW in this paper. While other studies from India [11] and abroad [2729] have included a longer time-frame (1-year or ever) for having had sex with female to label a person as MSMW or MSMO, we used a relatively shorter timeframe of one month as we did not have data on whether the participants had sex with women in the previous 6 months or 1 year.

Another major limitation is the lack of explicit timeframe for measuring consistency of condom use, even though the responses were interpreted to be consistent condom use in the recent past. Similarly, timeframes for reporting the types of male and female partners were slightly different, although for practical considerations, sexual partnerships were considered as concurrent, if the partnership was mentioned as current (e.g., spouse or female regular partner) or within the past month, as the eligibility criteria was that the participant must have had sex with a man in the past month. As sensitive information about sexual behaviors and condom use were asked, social desirability might have affected the responses. While the association between frequency of alcohol use and condom use was examined in this paper, this study did not collect information on alcohol use before having sex. However, other studies among MSM in India [23, 24, 30] have reported that alcohol use before having anal sex was associated with lack of condom use. Where relevant, future quantitative studies on sexual risk (condom use) among MSM need to collect data on alcohol use before having sex.

\section{Implications}

Like other studies from India, this study found bisexual behavior across all subgroups of MSM, including kothiidentified MSM. This means that assumptions regarding one's sexual behavior or marital status should not be based on self-reported sexual identity alone. This has important implications for clinical and counselling practice in terms on asking the sexual history in a sensitive manner and providing appropriate safer sex information. A significant proportion of MSMW inconsistently use condoms with both male and female partners, therefore it is critical that HIV interventions specifically address the need to consistently use condoms with partners of either gender; and provide tailored support in using condoms with all types of female partners, especially female regular partners; and to encourage MSM in getting their female regular partners tested for HIV or STIs. Support also needs to be available to MSMW who wish to disclose their same-sex sexual behavior to female regular partners, as disclosure might possibly help in practicing safer-sex with female regular partners. Diversity in sexual behavior of self-identified MSM needs to be explicitly discussed with all subgroups of MSM and stigma associated with heterosexual marriage within the self-identified MSM communities needs be addressed so that MSM who are married or have female partners could then openly discuss the challenges they have in practicing safer sex with their female partners. Future research can specifically study bisexual concurrency with specified timeframes for different types of male and female partners and consistency in condom use, and also examine the prevalence of HIV and condom use in anal sex with different types of female partners. Bisexual behavior and bisexual concurrency (sex with partners of both genders, who may be regular, casual, paying or paid partners) among heterosexual-identified men too need to be studied to assess their HIV-related risk behaviors and to compare the differences between heterosexually-identified MSMW and non-heterosexually identified MSMW, using both quantitative and qualitative methods.

\section{Conclusion}

Bisexual partnerships are commonly reported among diverse subgroups of self-identified MSM (such as kothis and double-deckers) in India. Our study found high HIV prevalence among both MSMW and MSMO, but it did not differ significantly between these two groups, after controlling for HIV intervention exposure. Also, MSMW, in general, were found to have high levels of inconsistent condom use with both male and female partners. HIV interventions among MSM need to acknowledge bisexual behavior among even self-identified MSM, and educate and counsel them on the risks associated with both unprotected anal and vaginal sex, and provide support for consistent use of condoms with partners of either gender. Married MSM also need to be trained on practical sexual communication and negotiation skills in dealing with the stigma surrounding condom use with spouse (which is seen as a sign of mistrust and infidelity). In addition, steps need 
to be taken to decrease the stigma faced by heterosexuallymarried self-identified MSM so that married MSM could access HIV-related services from community organizations as well as receive the necessary psychosocial support from their peers. Innovative ways of screening and treating female partners of self-identified MSM for HIV/STI, while maintaining the confidentiality of the sexuality of their husbands, need to be developed to decrease the risk of HIV/STI transmission and acquisition.

Acknowledgments The Bill \& Melinda Gates Foundation funded this research through Avahan: the India AIDS Initiative. The views expressed herein are those of the authors and do not necessarily reflect the official policy or position of the foundation and Avahan. The authors wish to thank Dr. Steve Mills from FHI 360, Asia Pacific Regional Office, Bangkok, Thailand for his inputs in finalization of the manuscript. Finally we thank the MSM community for their participation in the study.

Open Access This article is distributed under the terms of the Creative Commons Attribution 4.0 International License (http:// creativecommons.org/licenses/by/4.0/), which permits unrestricted use, distribution, and reproduction in any medium, provided you give appropriate credit to the original author(s) and the source, provide a link to the Creative Commons license, and indicate if changes were made.

\section{References}

1. Beyrer C, Baral SD, van Griensven F, Goodreau SM, Chariyalertsak S, Wirtz AL, Brookmeyer R. Global epidemiology of HIV infection in men who have sex with men. Lancet. 2012;380(9839):367-77.

2. Department of AIDS Control MoHFW. Annual Report 2012-13. 2013.

3. Asthana S, Oostvogels R. The social construction of male 'homosexuality' in India: implications for HIV transmission and prevention. Soc Sci Med. 2001;52(5):707-21.

4. Boyce P. Conceiving kothis': men who have sex with men in India and the cultural subject of HIV prevention. Med Anthropol. 2007;26(2):175-208.

5. Chakrapani V, Newman PA, Mhaprolkar H, Kavi AR. Sexual and social networks of MSM and Hijras in India: a qualitative study. 2007.

6. Phillips AEBM, Lowndes CM, Garnett GP, Gurav K, Ramesh BM, Anthony J, Watts R, Moses S, Alary M. Sexual identity and its contribution to MSM risk behavior in Bangaluru (Bangalore), India: the results of a two-stage cluster sampling survey. J LGBT Health Res. 2008;4(2-3):111-26.

7. Chakrapani VNPA, Shunmugam M. Secondary HIV prevention among kothi-identified MSM in Chennai, India. Cult Health Sex. 2008;10(4):313-27.

8. Narayanana P, Das A, Morineau G, Prabhakar P, Deshpande GR, Gangakhedkar R, Risbud A. An exploration of elevated HIV and STI risk among male sex workers from India. BMC Public Health. 2013;3:1059.

9. Jha UMRY, Venkatesh S, Dhingra N, Paranjpe RS, Saggurti N. HIV epidemic among men who have sex with men in India: national scenario of an unfinished agenda. HIV AIDS. 2014;6:159-70.

10. Friedman MR, Kurtz SP, Buttram ME, Wei C, Silvestre AJ. Stall R (2014) HIV risk among substance-using men who have sex with men and women (MSMW): findings from South Florida. AIDS Behav. 2014;18(1):111-9.

11. Phillips AE, Lowndes CM, Boily MC, et al. Men who have sex with men and women in Bangalore, South India, and potential impact on the HIV epidemic. Sex Transm Infect. 2010;86(3):187-92.

12. Kumta S, Lurie M, Weitzen S, Jerajani H, Gogate A, Row-kavi A. Bisexuality, sexual risk taking, and HIV prevalence among men who have sex with men accessing voluntary counseling and testing services in Mumbai, India. J Acquir Immune Defic Syndr. 2010;53(2):227-33.

13. Brahmam GN, Kodavalla V, Rajkumar H, Rachakulla HK, Kallam S, Myakala SP, Paranjapeb RS, Guptec MD, Ramakrishnan L, Kohli A, Ramesh BM. Sexual practices, HIV and sexually transmitted infections among self-identified men who have sex with men in four high HIV prevalence states of India. AIDS. 2008;22(suppl 5):S45-57.

14. Setia MS, Sivasubramanian M, Anand V, Row-Kavi A, Jerajani HR. Married men who have sex with men: the bridge to HIV prevention in Mumbai. India. Int J Public Health. 2010;55(6): 687-91.

15. International FH. Behavioral surveillance surveys (BSS): guidelines for repeated behavioral surveys in populations at risk of HIV. 2000.

16. Saidel T, Adhikary R, Mainkar M, et al. Baseline integrated behavioural and biological assessment among most at-risk populations in six high-prevalence states of India: design and implementation challenges. AIDS. 2008;22(Suppl 5):S17-34.

17. National AIDS Control Organisation (NACO) MoHFW. Targeted interventions under NACP III: operational guidelines, vol. 1. In: NACO, editor; 2007.

18. Go VF, Srikrishnan AK, Sivaram S, et al. High HIV prevalence and risk behaviors in men who have sex with men in Chennai, India. J Acquir Immune Defic Syndr. 2004;35(3):314-9.

19. Yadav D, Chakrapani V, Goswami P, Ramanathan S, Ramakrishnan L, George B, et al. Association between alcohol use and HIV-related sexual risk behaviors among men who have sex with men (MSM): findings from a multi-site bio-behavioral survey in India. AIDS Behav. 2014;18(7):1330-8.

20. Boyce P, Chakrapani V, Dhanikachalam D. Hard-to-reach men who have sex with men in India: Recommendations for HIV prevention. India 'MSM situation paper' series-Technical Brief 1. Futures Group International; 2011.

21. Chakrapani V, Boyce P, Dhanikachalam D. Women partners of men who have sex with men in India. India 'MSM situation paper' series-Technical Brief 2. Futures Group International; 2011.

22. Beyrer C, Trapence G, Motimedi F, et al. Bisexual concurrency, bisexual partnerships, and HIV among Southern African men who have sex with men. Sex Transm Infect. 2010;86(4):323-7.

23. Chakrapani V, Boyce P, Newman PA, Row Kavi A. Contextual influences on condom use among men who have sex with men in India: subjectivities, practices and risks. Cult Health Sex. 2013;15(8):938-51.

24. Chakrapani V, Newman PA, Shunmugam M. Secondary HIV prevention among kothi-identified MSM in Chennai, India. Culture, Health and Sexuality. 2008;10(4):313-27.

25. Pandya A, Pandya S, Patil B, Merchant S. Invisible "others": sexual health vulnerabilities of wives of men who have sex with men. Sex Cult. 2012;16(1):76-89.

26. Solomon SS, Mehta SH, Latimore A, Srikrishnan AK, Celentano DD. The impact of HIV and high-risk behaviours on the wives of married men who have sex with men and injection drug users: implications for HIV prevention. J Int AIDS Soc. 2010;13(Suppl 2):S7.

27. Maulsby CSF, German D, Flynn CP, Holtgrave D. HIV risk among men who have sex with men only (MSMO) and men who 
have sex with men and women (MSMW) in Baltimore. J Homosex. 2013;60(1):51-68.

28. Gorbach PMMR, Weiss RE, Hucks-Ortiz C, Shoptaw S. Bridging sexual boundaries: men who have sex with men and women in a street-based sample in Los Angeles. J Urban Health. 2009;86(1): S63-76.

29. Liao M, Kang D, Jiang B, et al. Bisexual behavior and infection with HIV and syphilis among men who have sex with men along the east coast of China. AIDS Patient Care STDS. 2011;25(11): 683-91.

30. Shunmugam M, Sivasubramanian, M, Roy Karmakar S, Samuel M, Chakrapani V. Extent of alcohol use and its association with inconsistent condom use among hijras/transgender (male-to-female) people in India: survey findings from urban and rural sites in Maharashtra and Tamil Nadu. Paper Presented at International AIDS Conference, Washington DC, USA; 2012. 